\title{
Industry-Oriented Method for Dynamic Force Identification in Peripheral Milling Based on FSC- LSQR Using Acceleration Signals
}

\section{Maxiao Hou}

Xi'an Jiaotong University

Hongrui Cao ( $\square$ chr@mail.xjtu.edu.cn )

Xi'an Jiaotong University https://orcid.org/0000-0002-2001-8131

Qi Li

Xi'an Jiaotong University

Jianghai Shi

Xi'an Jiaotong University

\section{Research Article}

Keywords: Peripheral milling, Dynamic force identification, Queue buffer structure.

Posted Date: January 7th, 2022

DOI: https://doi.org/10.21203/rs.3.rs-1218168/v1

License: (a) (i) This work is licensed under a Creative Commons Attribution 4.0 International License. Read Full License 


\title{
Industry-oriented Method for Dynamic Force Identification in Peripheral Milling Based on FSC-LSQR Using Acceleration Signals
}

\author{
Maxiao Hou ${ }^{1}$, Hongrui $\mathrm{Cao}^{1 *}$, Qi $\mathrm{Li}^{1}$ and Jianghai $\mathrm{Shi}^{1}$ \\ ${ }^{1}$ State Key Laboratory for Manufacturing Systems Engineering, \\ Xi'an Jiaotong University, Xi'an, 710049, China.
}

*Corresponding author(s). E-mail(s): chr@mail.xjtu.edu.cn; Contributing authors: houmax@stu.xjtu.edu.cn; li_qi_1996@163.com; shi_jianghai@163.com;

\begin{abstract}
Online measurement of milling force play a vital role in enabling machining process monitoring and control. In practice, the milling force is difficult to be measured directly with the dynamometer. This paper develops a novel method for milling force identification called least square QR-factorization with fast stopping criterion (FSC-LSQR) method, and the queue buffer structure (QBS) is employed for the online identification of milling force using acceleration signals. The convolution integral of milling force and acceleration signals is discretized, which turns the problem of milling force identification into a linear discrete ill-posed problem. The FSC-LSQR algorithm is adopted for milling force identification because of its high efficiency and accuracy, which handles the linear discrete ill-posed problem effectively. The online identification of milling force can be realized using the acceleration signal enqueue and the milling force dequeue operations of the QBS. Finally, the effectiveness of the method is verified by experiments. The experimental results show that the FSC-LSQR algorithm running time is within $\mathbf{0 . 0 5 s}$ and the calculation error is less than $\mathbf{1 0 \%}$. The proposed method can make the sampling frequency of the milling force reach $10240 \mathrm{~Hz}$ by employing QBS, which satisfy the industry requirements of milling force measurement.
\end{abstract}

Keywords: Peripheral milling, Dynamic force identification, Queue buffer structure. 


\section{Introduction}

Milling has been of a great important in the field of machining process and the impact cannot be overemphasized. It is widely used in national defense, aviation, aerospace, and other fields. Since the goals of the milling process are to achieve high productivity and better surface quality, process disturbances like chatter, collision, and tool wear need to be monitored and controlled [1, 2]. All studies on these problems are closely related to milling forces. Therefore, becoming vital for the intelligent spindle to sense the milling force in real time.

The piezoelectric dynamometer is the most commonly used instrument for online measurement of milling force [3, 4]. The milling force measurement of rotating tools still has many limitations in industrial applications: (1) the dynamometer is too expensive; (2) the workpiece size is greatly limited; (3) inconvenient installation of the dynamometer. In order to overcome these shortcomings, Rizal et al. [5] proposed to install a force sensor between the spindle and the tool to measure the milling force, and verified its effectiveness through experiments. Kistler corporation has developed the wireless rotary milling force measurement instrument. The rotary dynamometer mentioned above is extremely expensive and complicated in structure, which means that the dynamometer can just be used in laboratories rather than the actual milling process. In summary, there is an urgent need for a low-cost, easy-to-install, fast, and accurate milling force measurement system.

In recent years, many experts thought of using current, displacement, and other signals to identify milling forces. Machine tool feed motor contains a large amount of processing information. Using the feed motor current signal, Jeong et al. [6] and Aslan et al. [7] identify the milling force and extend its identification bandwidth; however, this method requires accurate system parameters. Kim et al. [8], Chen et al. [9] and Song et al. [10] choose the spindle motor current with higher static quasi-state sensitivity to monitor the milling force. The frequency bandwidth of the milling forces identification using current signal is relatively narrow [11]. Kim et al. [12, 13] proposed a method of using a cylindrical capacitive displacement sensor to quantitatively estimate the milling force. Albrecht et al. [14] used the Kalman filter to reduce the influence of unknown disturbance and measurement error on the identification of milling force by displacement signals. Multi-sensor fusion technology is used to improve the accuracy of milling force identification, such as displacement and acceleration (Zhou et al. [15] and Salehi et al. [16]), current and acceleration (Hamid et al. [17]). Although the method above can identify the milling force in the highspeed milling process, the increase in cost and the decrease in reliability are inevitable.

Compared with the displacement sensor, the accelerometer has the advantages of easier installation and lower cost, which makes the acceleration signal more suitable for identifying the milling force in the actual milling process. For the fisrt time, an indirect force estimation method from accelerometers mounted on the spindle housing is proposed [18]. In the actual industrial environment, it is difficult to measure the frequency response function of the spindle 
under rotating conditions, even if this modal characterization is more accurate. So the impact hammer experiment is used to obtain the frequency response function of the spindle. Both the acceleration signal and the spindle frequency response function can be measured. It seems that the milling force can be identified by using the inverse filtering method [19]. However [20] pointed out that the inverse filtering method is sensitive to noise at certain frequencies, and even small noises will have large errors. In actual milling conditions, the transfer matrix between acceleration signals and milling force is usually illconditioned and cannot be directly inverted to identify the milling force. This kind of problem is called linear discrete ill-posed problem in the field of mathematics. Regularization is the main method for solving linear discrete ill-posed problems, including direct regularization algorithm and iterative regularization algorithm. The most common direct regularization algorithms (Tikhonov [21] and TSVD [22]) are introduced to identify the milling force by measuring acceleration signals. Compared with direct regularization algorithms, iterative regularization algorithms can reduce the amount of calculation and quickly obtain the approximate solution when solving linear discrete ill-posed problems. Especially for solving large-scale problems, iterative regularization algorithms will have more advantages. Wang et al. [23] introduced a conjugate gradient least square (CGLS) algorithm to achieve identification of milling force by using filtered acceleration signals. Morigi et al. [24] have demonstrated that the LSQR algorithm has better numerical stability than the CGLS algorithm. The iteration stopping criterion of the LSQR algorithm often requires reference value or high time cost, which makes the traditional LSQR algorithm can only identify the milling force in theory. Up to now, the indirect identification method of milling force has been implemented under offline conditions, and no one has given the process of how to use the proposed method to realize the online identification of milling force in the actual milling process.

The purpose of this paper is to realize online identification of dynamic milling force by using acceleration signals and to provide an industry-oriented milling force measurement method. The proposed FSC-LSQR algorithm overcomes the excessive number of iterations and inaccurate results caused by the Semi-convergence of the LSQR algorithm solution. Based on the FSC-LSQR algorithm, the online identification of milling force in the actual milling process is realized by using the QBS, which gives the idea of online identification of milling force. Finally, the milling experiments validate the effectiveness and generalization of the proposed method. In addition, the CGLS algorithm [23] is also implemented for comparison, which shows that the FSC-LSQR algorithm has higher identification speed and accuracy.

\section{Problem statement of milling force identification}

The milling process of the three-flute milling cutter is shown in Figure 1. Take the tool tip position as the origin, the milling feed direction as the $\mathrm{x}$ 
axis, and the direction perpendicular to the $\mathrm{x}$ axis as the $\mathrm{y}$ axis to establish a two-degree-of-freedom rectangular coordinate system.

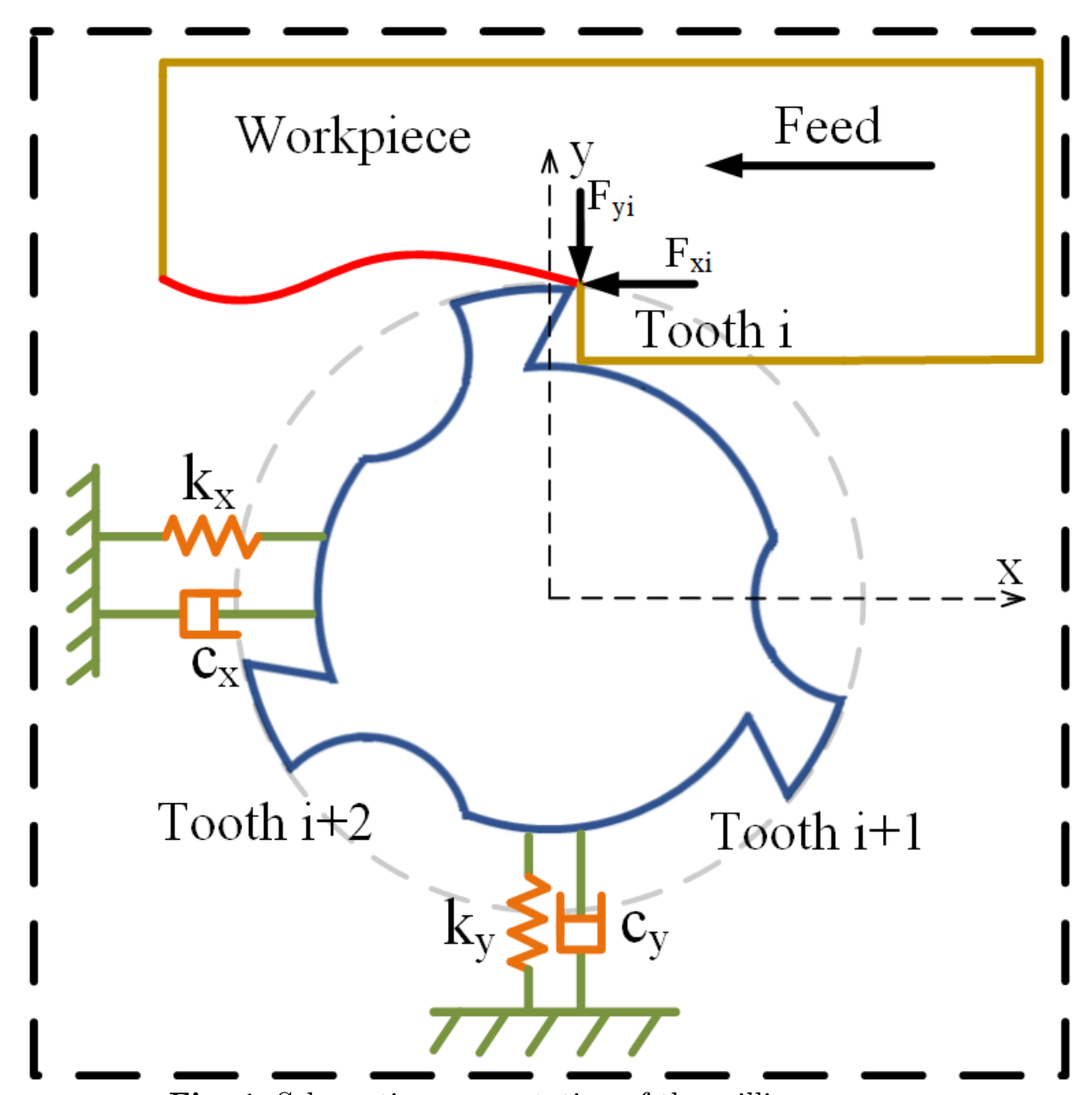

Fig. 1: Schematic representation of the milling process.

The two-degree-of-freedom equation of motion of the milling process is given as follows:

$$
\boldsymbol{M} \ddot{\boldsymbol{Z}}(t)+\boldsymbol{C} \dot{\boldsymbol{Z}}(t)+\boldsymbol{K} \boldsymbol{Z}(t)=\boldsymbol{F}(t)
$$

where $\boldsymbol{M}, \boldsymbol{C}$ and $\boldsymbol{K}$ are $2 \times 2$ mass, stiffness and damping matrices, respectively. $\boldsymbol{Z}(t)=[x(t) y(t)]^{T}, x(t)$ and $y(t)$ are tool displacements in the feed $(\mathrm{x})$ and cross-feed $(\mathrm{y})$ directions, $\boldsymbol{F}(t)=\left[F_{x}(t) F_{y}(t)\right]^{T}, F_{x}(t)$ and $F_{y}(t)$ are milling forces in the $\mathrm{x}$ and $\mathrm{y}$ directions. 
Since the $\mathrm{x}(\mathrm{y})$ direction excitation has much less influence on the $\mathrm{y}(\mathrm{x})$ direction response of the spindle system than the same magnitude excitation in the $\mathrm{x}(\mathrm{y})$ direction, the cross effect between the $\mathrm{x}$ and $\mathrm{y}$ directions of the spindle system is not considered, that is, $\boldsymbol{M}=\left[\begin{array}{cc}m_{x} & 0 \\ 0 & m_{y}\end{array}\right], \boldsymbol{C}=\left[\begin{array}{cc}c_{x} & 0 \\ 0 & c_{y}\end{array}\right]$ and $\boldsymbol{K}=\left[\begin{array}{cc}k_{x} & 0 \\ 0 & k_{y}\end{array}\right]$

Taking the $\mathrm{x}$ direction as an example, the response $x(t)$ under the milling force $F_{x}(t)$ can be calculated by Duhamel integration [25] as follows:

$$
x(t)=m_{x}\left(\dot{x}_{0}+2 \delta_{x} \omega_{x} x_{0}\right) h_{x x}(t)+m_{x} x_{0} \dot{h}_{x x}(t)+\int_{0}^{t} h_{x x}(t-\tau) F_{x}(\tau) d \tau
$$

where $x_{0}$ is the initial value of the displacement, $m_{x}$ is modal mass, $\delta_{x}$ is damping ratio, $\omega_{x}$ is natural frequency, $h_{x x}(t)$ is the transfer function.

According to [23], the equation (2) can be omitted as follows:

$$
x(t)=\int_{0}^{t} h_{x x}(t-\tau) F_{x}(\tau) d \tau
$$

The $x(t)$ in equation (3) has a general definition, it can be displacement, velocity, or acceleration, and the same as $h_{x x}(t)$ also corresponds to the displacement transfer function, velocity transfer function or acceleration transfer function. Accelerometer has been the most widely used in industrial environments. Therefore, the acceleration signal is used to identify the milling force in the actual milling process.

The equation (3) can be discretized into a linear algebraic equation as shown below.

$$
\begin{aligned}
& {\left[\begin{array}{c}
x(\Delta t) \\
x(2 \Delta t) \\
\vdots \\
x((n-1) \Delta t) \\
x(n \Delta t)
\end{array}\right]=\Delta t\left[\begin{array}{cc}
h_{x x}(\Delta t) & 0 \\
h_{x x}(2 \Delta t) & h_{x x}(\Delta t) \\
\vdots & \vdots \\
h_{x x}((n-1) \Delta t) & h_{x x}((n-2) \Delta t) \\
h_{x x}(n \Delta t) & h_{x x}((n-1) \Delta t)
\end{array}\right.} \\
& \left.\begin{array}{ccc}
\cdots & 0 & 0 \\
\cdots & 0 & 0 \\
\ddots & \vdots & \vdots \\
\cdots & h_{x x}(\Delta t) & 0 \\
\cdots & h_{x x}(2 \Delta t) & h_{x x}(\Delta t)
\end{array}\right]\left[\begin{array}{c}
F_{x}(\Delta t) \\
F_{x}(2 \Delta t) \\
\vdots \\
F_{x}((n-1) \Delta t) \\
F_{x}(n \Delta t)
\end{array}\right]
\end{aligned}
$$

where $\Delta t=t / n$ is the time interval, $n$ is the sampling length. 
Equation (4) can be simplified to the following form

$$
H_{x x} F_{x}=x
$$

where $x=x(t)$ is the measured acceleration signal, the matrix $H_{x x}$ represents the dynamic characteristics of the system.

It can be seen from equation (1) that the milling force includes two directions: $\mathrm{x}$ direction and $\mathrm{y}$ direction. According to equation (5), the following equation can be obtained.

$$
\left[\begin{array}{cc}
H_{x x} & 0 \\
0 & H_{y y}
\end{array}\right]\left[\begin{array}{l}
F_{x} \\
F_{y}
\end{array}\right]=\left[\begin{array}{l}
x \\
y
\end{array}\right]
$$

where $y, H_{y y}$ and $F_{y}$ are similar to the derivation of $x, H_{x x}$ and $F_{x}$ in equation (5).

The equation (6) is simplified to the following form

$$
\boldsymbol{H} \boldsymbol{F}=\boldsymbol{X}
$$

where $\boldsymbol{H}=\left[\begin{array}{cc}H_{x x} & 0 \\ 0 & H_{y y}\end{array}\right], \boldsymbol{F}=\left[\begin{array}{c}F_{x} \\ F_{y}\end{array}\right], \boldsymbol{X}=\left[\begin{array}{l}x \\ y\end{array}\right]$.

It can be seen from equation (7) that the acceleration signal can be used to identify the milling force only by inverting the matrix $\boldsymbol{H}$, and the equation is obtained as follows:

$$
\boldsymbol{F}=\boldsymbol{H}^{-1} \boldsymbol{X}
$$

From the equation (8), it seems easy to identify the milling force by using acceleration signals. However, in the actual milling process, the unknown disturbance is inevitable, and the obtained matrix $\boldsymbol{H}$ is an ill-conditioned matrix that usually satisfy the following three conditions:

(1) The singular value gradually decays to zero;

(2) The number of conditions is large;

(3) Discrete Picard condition is satisfied.

According to the characteristics of matrix $\boldsymbol{H}$, the identification of milling force is a linear discrete ill-posed problem. We cannot solve equation (8) directly. Instead, a feasible method is used to find the approximate solution of $\boldsymbol{F}$ as the numerical solution of equation (8).

The core problems to be solved are summarized as follows:

Problem: How to efficiently and accurately solve the approximate solution of the milling force by using the measured acceleration signal, so as to meet the requirements of milling force measurement in the actual milling process. 


\section{Online identification method of milling force}

In the actual milling process, the accelerometers in the feed and cross-feed directions transmit the acceleration signals to the computer through the driver and DAQ-board. The computer uses the QBS to realize the online identification of the milling force based on the proposed FSC-LSQR algorithm. The specific process is shown in Figure 2.

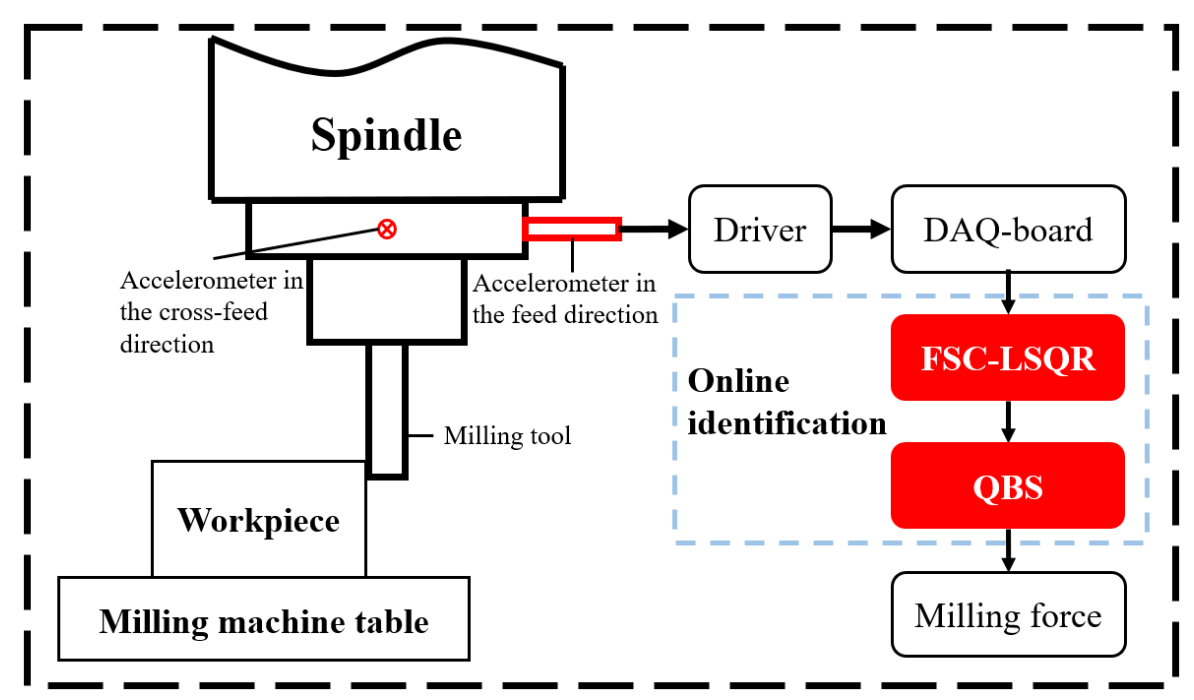

Fig. 2: The process of online identification of milling force by using acceleration signals.

\subsection{Theory of fast stopping criterion for least square QR-factorization algorithm}

In order to solve the core problem, the identification method of milling force is required to have a fast solution speed, that is, to efficiently and accurately solve the approximate solution of the milling force $\boldsymbol{F}$ in the equation (7). We refer to the discussion and analysis of a series of related iterative algorithms by Meurant [26], and choose one of the most commonly used and most wellknown algorithms, the LSQR algorithm. Based on the LSQR algorithm, a fast stopping criterion that satisfy the practical industrial application is given, which can quickly and accurately identification of milling force. The LSQR algorithm is described in [27].

When the LSQR algorithm is used to identify the milling force, the initial value of the approximate solution of the milling force is the zero vector $\boldsymbol{F}_{0}=\mathbf{0}$. The approximate solution $\boldsymbol{F}_{k}$ for the milling force $\boldsymbol{F}$ satisfies the following 
properties.

$$
\left\|\boldsymbol{H} \boldsymbol{F}_{k}-\boldsymbol{X}\right\|=\min _{\boldsymbol{F} \in K_{k}\left(\boldsymbol{H}^{T} \boldsymbol{H}, \boldsymbol{H}^{T} \boldsymbol{X}\right)}\|\boldsymbol{H} \boldsymbol{F}-\boldsymbol{X}\|
$$

where $K_{k}\left(\boldsymbol{H}^{T} \boldsymbol{H}, \boldsymbol{H}^{T} \boldsymbol{X}\right)=\operatorname{span}\left\{\boldsymbol{H}^{T} \boldsymbol{X},\left(\boldsymbol{H}^{T} \boldsymbol{H}\right) \boldsymbol{H}^{T} \boldsymbol{X}, \ldots,\left(\boldsymbol{H}^{T} \boldsymbol{H}\right)^{k-1} \boldsymbol{H}^{T} \boldsymbol{X}\right\}$ represents the Krylov subspace, span $\{\cdot\}$ is the vector space.

It can be seen from equation (9) that the LSQR algorithm is essential to minimizing the residuals. At the $k$-th step, $\boldsymbol{F}_{k}$ is searched to minimize the euclidean norm of the residual $\boldsymbol{r}_{k}=\boldsymbol{H} \boldsymbol{F}_{k}-\boldsymbol{X}$. According to the properties of the LSQR algorithm, the residual satisfies the following inequality.

$$
\left\|\boldsymbol{r}_{k-1}\right\| \geq\left\|\boldsymbol{r}_{k}\right\| \quad k=1,2, \ldots, N .
$$

The inequality (10) is strictly established.

According to inequality (10), it can only guarantee that the residual norm of the obtained approximate solution decreases monotonically as the number of iteration steps $k$ increases. We cannot guarantee that the error norm $\left\|\boldsymbol{F}_{k}-\boldsymbol{F}\right\|$ will also continue to decrease. Normally, the error norm does gradually decrease in the initial few iteration steps, but after it is reduced to a certain level, the value of the error norm begins to increase as the number of iteration steps $k$ further increases. This property exhibited by the iterative solution $\boldsymbol{F}_{k}$ is called semi-convergence [28]. The principle of the LSQR algorithm for the approximate solution is analyzed above. The stop iteration criterion of the LSQR algorithm is only to find the minimum residual $\boldsymbol{r}_{k}$, the approximate solution obtained $\boldsymbol{F}_{k}$ is inaccurate and the calculation time will increase as the number of iteration steps $k$ increases. The LSQR algorithm cannot meet the requirements of online identification of milling force in the industry. We try to make the LSQR algorithm stop the iteration in time when its iterative solution error norm $\left\|\boldsymbol{F}_{k}-\boldsymbol{F}\right\|$ is as small as possible.

The fast stopping criterion for the LSQR iterative algorithm is considered. The goal is to make the LSQR algorithm iteratively stop in time and obtain the accurate estimate of the milling force as possible. We use the properties of the iterative solutions obtained by two iterative algorithms (i.e. LSQR and Craig) to determine the stopping criterion of the LSQR algorithm and the approximate solution $F_{k}$ of the milling force. The specific method is to determine the stopping criterion and the corresponding iterative solution by comparing the residual $\boldsymbol{r}_{k}$ of the iterative solution obtained by the LSQR algorithm with the residual $\hat{\boldsymbol{r}}_{k}$ of the iterative solution obtained by the Craig algorithm. The property satisfied by the iterative solution of the Craig algorithm is different from the property of the LSQR algorithm.

$$
\left\|\hat{\boldsymbol{F}}_{k}-\boldsymbol{H}^{-1} X\right\|=\min _{\boldsymbol{F} \in K_{k}\left(\boldsymbol{H}^{T} \boldsymbol{H}, \boldsymbol{H}^{T} \boldsymbol{X}\right)}\left\|\boldsymbol{F}-\boldsymbol{H}^{-1} X\right\|
$$


where $\hat{\boldsymbol{F}}_{k}$ represents the approximate solution of the milling force obtained by the Craig algorithm.

The purpose of the Craig algorithm is not to minimize residuals, but to minimize errors, which is not easy to describe and measure in practical application. Although we will not simply use the Craig algorithm to solve ill-posed problems, the residual $\hat{\boldsymbol{r}}_{k}=\boldsymbol{H} \hat{\boldsymbol{F}}_{k}-\boldsymbol{X}$ obtained by the Craig algorithm is very helpful to better select the stopping criterion of the LSQR algorithm.

An industry-oriented FSC-LSQR algorithm is given to realize the online identification of milling force by using acceleration signals. The proposed FSCLSQR algorithm is based on the Golub-Kahan bidiagonalization, and runs the LSQR and the Craig algorithms at the same time. As the iteration proceeds, We can get the residual $\boldsymbol{r}_{k}$ of LSQR algorithm and the residual $\hat{\boldsymbol{r}}_{k}$ of Craig algorithm, and then use $\left\|\boldsymbol{r}_{k}\right\|$ and $\left\|\hat{\boldsymbol{r}}_{k}\right\|$ to determine when to stop the iterative of the LSQR algorithm.

Remark 1: The specific value of $\delta$ in the FSC-LSQR algorithm needs to be determined according to different industrial environments. FSC-LSQR algorithm introduces the Craig algorithm on the basis of the LSQR algorithm. The fast stopping criterion is given by the residual ratio of the Craig algorithm residual $\left\|\hat{\boldsymbol{r}}_{k}\right\|$ to the LSQR algorithm residual $\left\|\boldsymbol{r}_{k}\right\|$, which makes the milling force identification more accurate and fewer iterations. Both the LSQR algorithm and the Craig algorithm are based on the Golub-Kahan bidiagonalization process [24]. The FSC-LSQR algorithm only needs to do one vector product of matrices $\boldsymbol{H}$ and $\boldsymbol{H}^{T}$ in each iteration of the algorithm. Therefore, the calculation amount required for each iteration of the FSC-LSQR algorithm is essentially the same as that of the LSQR algorithm.

\subsection{Online identification strategy of milling force}

We use the QBS to show the whole process of the online identification of milling force in the actual milling process based on the FSC-LSQR algorithm, which is not available in the previous paper. In addition, the minimum requirement for the speed of the identification method is given. Only when the identification speed is higher than this requirement, then the online identification of milling force can be realized.

In order to realize the online identification of milling force, a storage structure that can output the identified milling force while inputting the acceleration signal is needed. The QBS in the data structure has the characteristics of first in first out (FIFO) [29], and then it is applied to online identification of milling force. Taking the QBS containing twenty rotation cycles as an example, the strategy for online identification of milling force in the actual milling process is given as shown in Figure 3. Where queue head pointer front and queue tail pointer rear are used to output milling force (dequeue) and input acceleration signal (enqueue), respectively. $\boldsymbol{F}_{i d_{j}}$ and $\boldsymbol{X}_{j}$ are the identified milling force and acceleration signal in the $j$-th cycle, $j=1, \ldots, n$.

It can be seen from Figure 3 that the queue with a length of twenty rotation cycles is given, where the first ten cycles are the identified milling force, and 


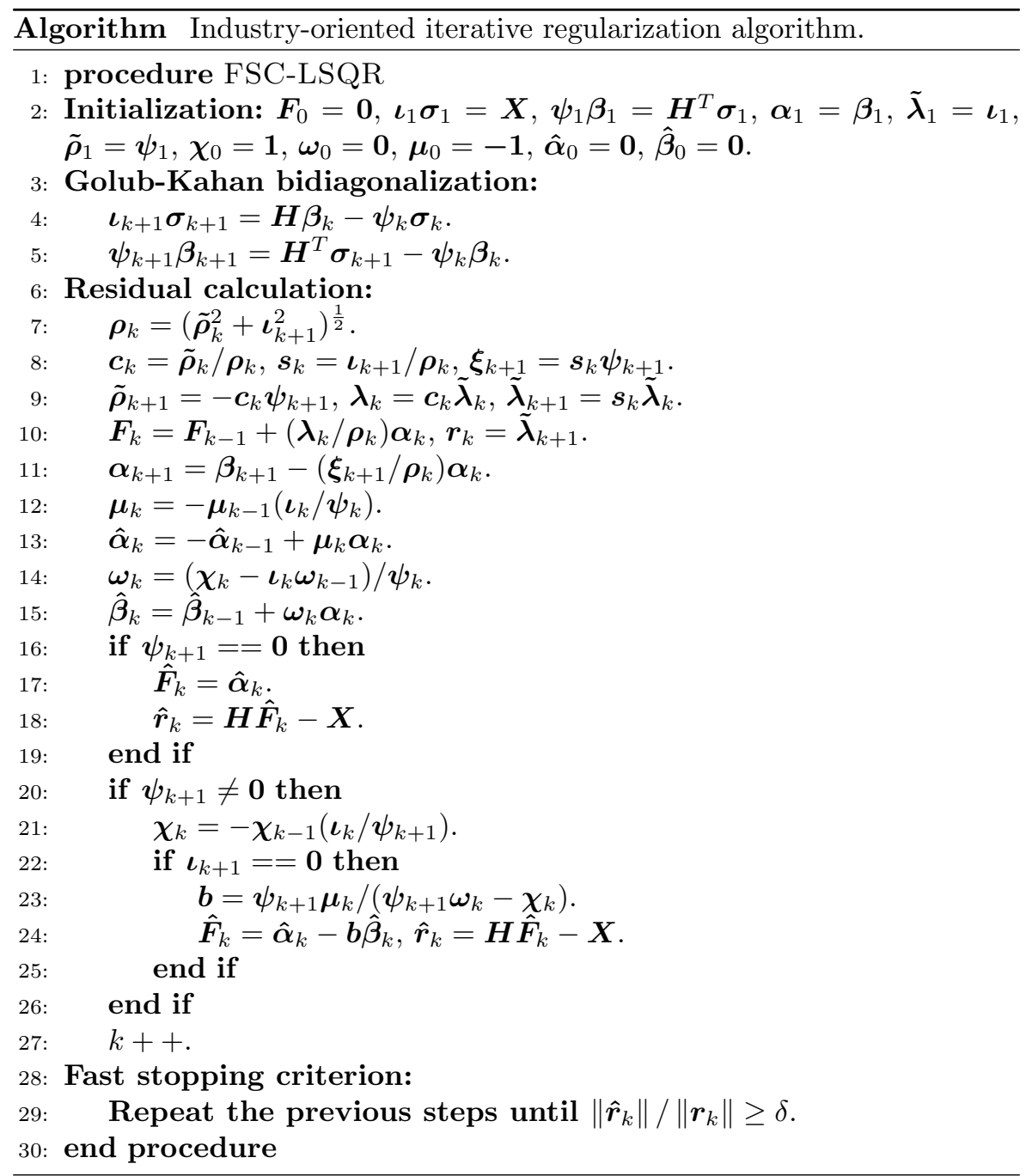

the last ten cycles are the acceleration signals. The milling force is dequeued in real time according to the sampling frequency of the acceleration signal, and the acceleration signal is enqueued at the same time. When the milling force of the tenth cycle is dequeued, the acceleration signal of the eleventh to the twentieth cycle has been identified as a milling force. In this way, the online identification of milling force can be realized. This strategy has a requirement, that is, when the queue length is twenty cycles, the identification time of ten cycles must be less than the output time of ten cycles. In other words, if the proposed method is not fast enough for milling force identification, it cannot meet the requirements of online identification of milling force. In the following experiments, it is verified that the proposed FSC-LSQR algorithm can output the milling force at a frequency of up to $10240 \mathrm{~Hz}$ in real time, 


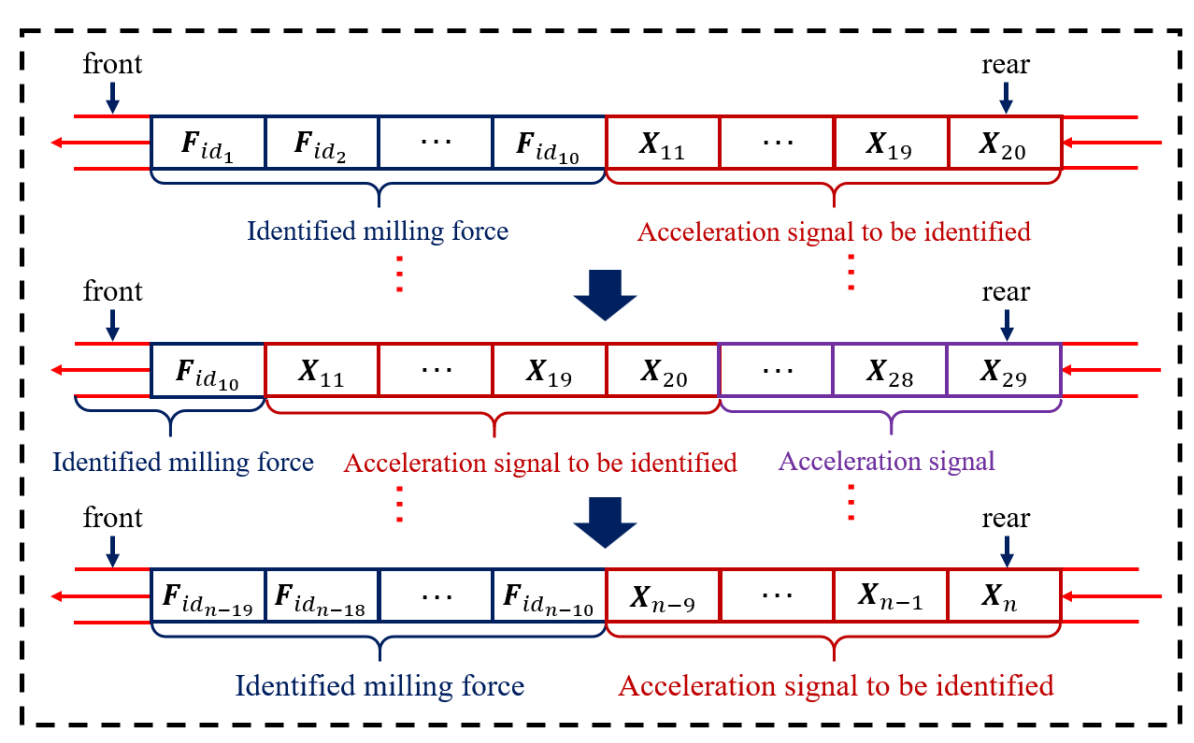

Fig. 3: The strategy for online identification of milling force.

which fully satisfy the requirements of industrial applications. Note that no matter what identification method of the milling force used, it takes time. The online identification strategy of the milling force will still have a time delay, which is unavoidable.

\section{Milling experiment for validation of the proposed method}

The experiment consists of two parts: the impact hammer experiment and the milling experiment. The equipment required for the experiment is shown in Figure 4. 

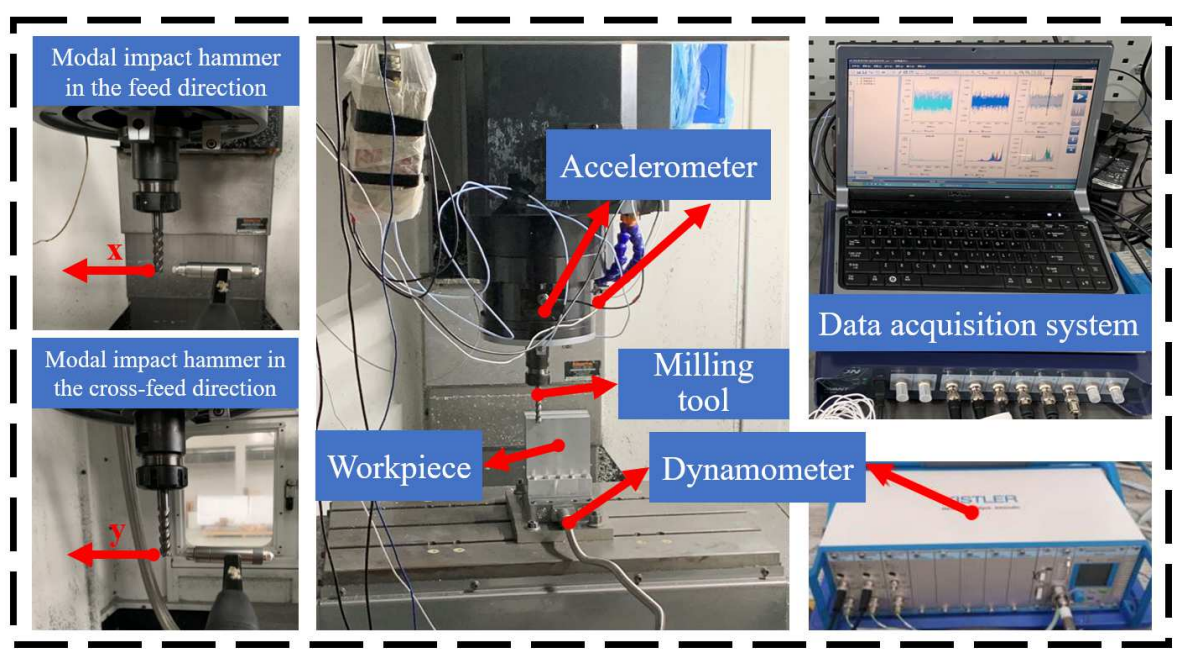

Fig. 4: Experiment setup.

As shown in Figure 4, the accelerometer is installed on the box close to the front bearing of the spindle along $\mathrm{x}$ and $\mathrm{y}$ directions. The installation position of the accelerometer minimizes the milling force transmission path, minimizes energy reduction, and has higher sensitivity. The dynamometer used in the experiment is the Kistler 9129A dynamometer, and the workpiece material is 6061 aluminum alloy. PCB-086C01 impact hammer (sensitivity is $2.25 \mathrm{mv} / \mathrm{N}$ ) is used to generate and measure excitation force, MI-7008 data acquisition system (sampling frequency is $10240 \mathrm{~Hz}$ ) is used to record excitation force and the response, and then output the frequency response function. Three-blade milling cutter (high-speed steel material, diameter $10 \mathrm{~mm}$, blade length $40 \mathrm{~mm}$, overhang length $60 \mathrm{~mm}$, helix angle $45^{\circ}$ ) is used. During the machining process, the spindle speed is $4000 \mathrm{r} / \mathrm{min}$, the milling depth is $1 \mathrm{~mm}$, the milling width is $3 \mathrm{~mm}$, and the feed rate is $480 \mathrm{~mm} / \mathrm{min}$.

\subsection{Frequency response function measurement}

The impact hammer experiment is carried out to obtain the acceleration frequency response function from the tool tip to the accelerometer. The acceleration frequency response functions (FRF- $x x$, FRF- $x y$, FRF-yy, FRF- $y x$ ) are obtained by averaging the acceleration frequency response function measured five times in the $x$ and $y$ directions. This method of averaging multiple experiments can reduce the influence of random errors, make the obtained frequency response functions more accurate. The acceleration frequency response functions obtained from the experiment are shown in Figure 5. 


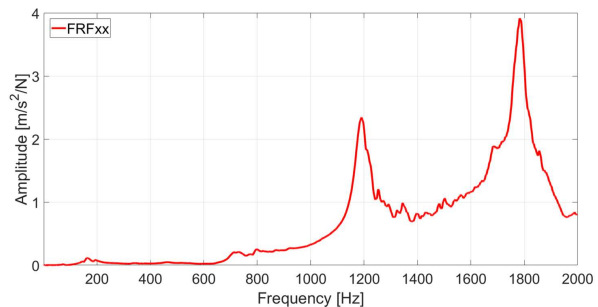

(a)

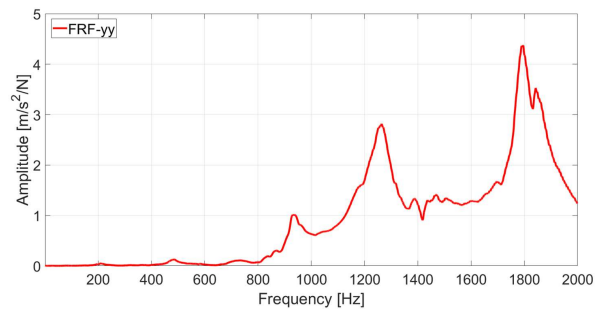

(c)

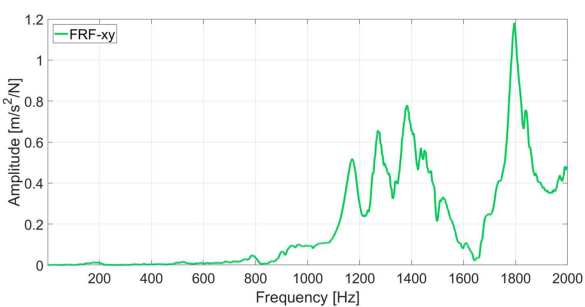

(b)

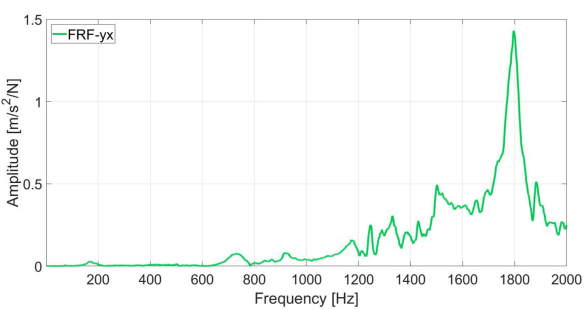

(d)

Fig. 5: Frequency response function of tool tip to accelerometer. (a) FRF- $x x$. (b) FRF-xy. (c) FRF-yy. (d) FRF-yx.

\subsection{Dynamic compensation of Kistler dynamometer}

The output value of the Kistler dynamometer is compensated, and then the compensated value is used as the reference standard for identifying milling force.

The reason why the output result of the Kistler dynamometer deviates from the actual milling force is explained. The milling force direct measurement system is shown in Figure 6, which is mainly composed of a workpiece, bolts, and a dynamometer. The workpiece and the dynamometer are connected by a total of ten bolts in the front and rear rows. The dynamometer is also fixed on the workbench by bolts. The milling force in the process of machining the workpiece surface which is measured by the Kistler dynamometer. 


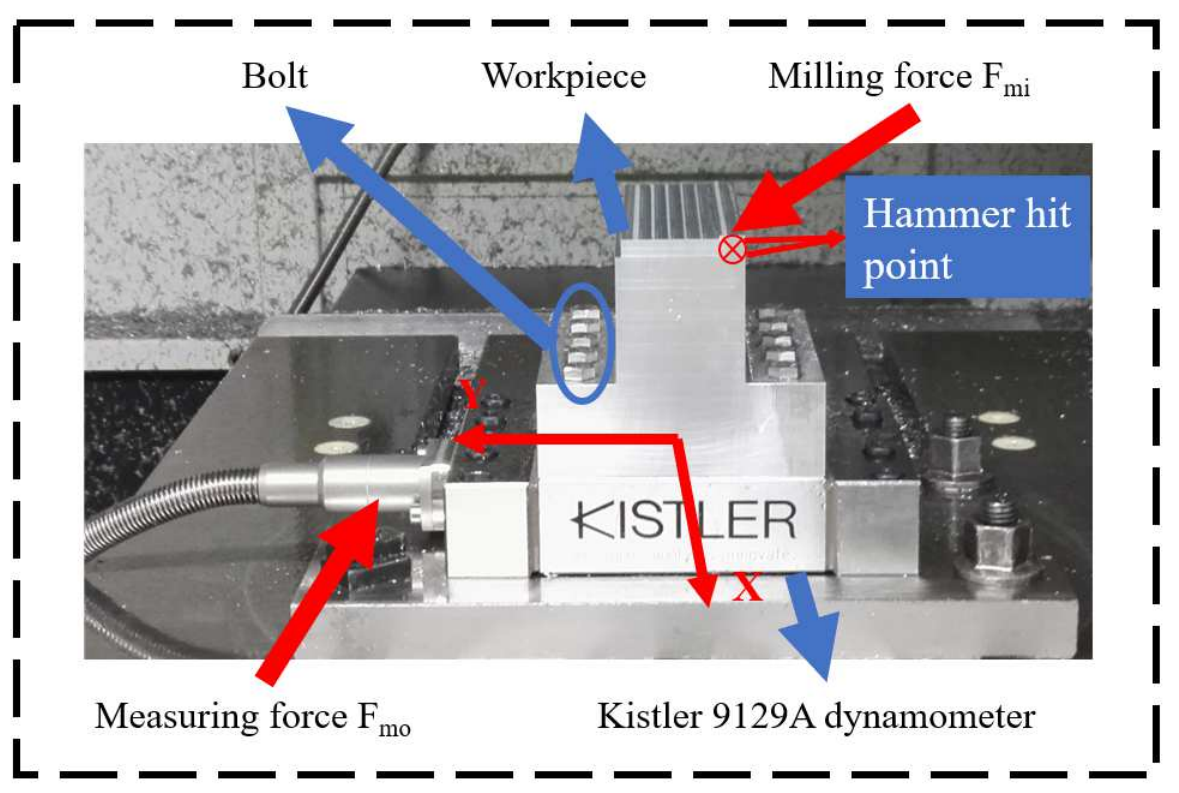

Fig. 6: Milling force measurement system.

The input and output of the milling force measurement system are $\boldsymbol{F}_{m i}$ and $\boldsymbol{F}_{m o}$ respectively. The relationship between $\boldsymbol{F}_{m i}$ and $\boldsymbol{F}_{m o}$ is given as follows:

$$
\boldsymbol{H}_{f}(\omega)=\frac{\boldsymbol{F}_{m o}(\omega)}{\boldsymbol{F}_{m i}(\omega)}
$$

where $\boldsymbol{H}_{f}(\omega)$ denote the frequency response function of measuring force and milling force, $\boldsymbol{F}_{m o}(\omega)$ denote the frequency domain representation of the measuring force $\boldsymbol{F}_{m o}, \boldsymbol{F}_{m i}(\omega)$ denote the frequency domain representation of the milling force $\boldsymbol{F}_{m i}$.

The average frequency response function of the system obtained through five impact hammer experiments is shown in Figure 7. Figures 7(a) and 7(b) correspond to the frequency response functions (FRF) in the feed (x) and crossfeed (y) directions, respectively. In ideal situations, the measurement result of the dynamometer is not affected by the dynamic characteristics of the milling force measurement system, that is, the amplitude of the frequency response function is 1 in the entire frequency band, but this is not the case in practice. As shown in Figure 7, when the frequency response $\mathrm{FRF}-x x$ and $\mathrm{FRF}-y y$ are in the range of $0-500 \mathrm{~Hz}$ and $0-550 \mathrm{~Hz}$, respectively, the amplitude of the frequency response function is close to 1 ; when the frequency is higher, the amplitude of the frequency response function is much greater than 1. Distortion of milling force measurement is not a unique phenomenon of the dynamometer. In fact, the influence of the dynamic characteristics of the milling force measurement 
system is widespread, so it is necessary to compensate for the measurement milling force results.

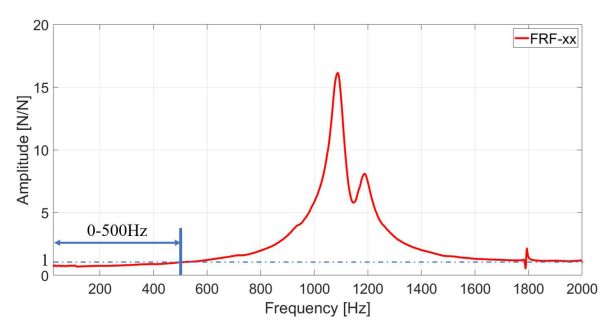

(a)

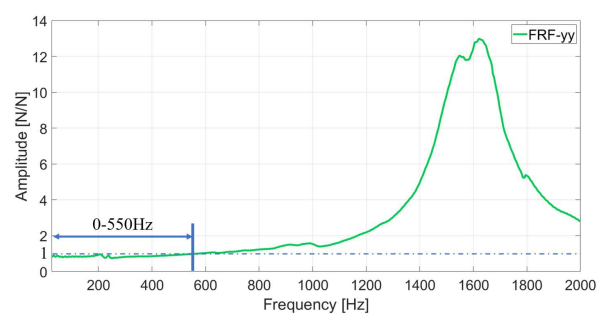

(b)

Fig. 7: Frequency response function of force measurement system. (a) FRF- $x x$. (b) FRF-yy.

Because the focus of this paper is not the dynamic compensation of the Kistler dynamometer, the improved inverse filter proposed by Wan et al. [30] is used to compensate the measured milling force. The compensated milling force is regarded as the actual milling force $\boldsymbol{F}$, which serves as a reference standard for verifying the accuracy of the identified milling force.

\subsection{Acceleration signal preprocessing}

Figures $8(\mathrm{a})$ and $8(\mathrm{~b})$ are the spectrum analysis results of the measured milling force after compensation in the $\mathrm{x}$ and $\mathrm{y}$ directions, respectively. It can be seen from Figures 8(a) and 8(b) that the frequency spectrum components of milling force are mainly distributed on the tooth passing frequency $f_{c}$ and its multipliers. Where $f_{c}=N \Omega / 60, \Omega$ is the spindle speed, and $N$ is the number of milling cutter teeth. In order to ensure the accuracy of the identification result, certain filtering processing of the acceleration signal is required. The Butterworth low-pass filter is selected here, and the cut-off frequency is $1500 \mathrm{~Hz}$ to eliminate the interference of high-frequency components while retaining the effective low-frequency components. 


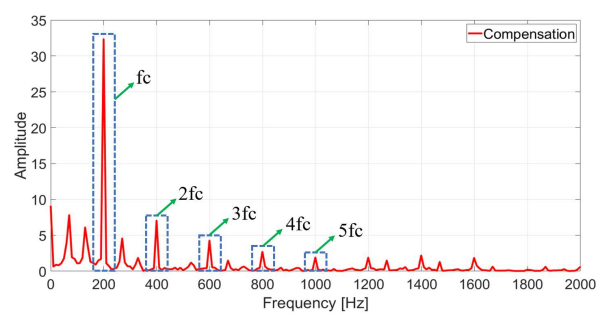

(a)

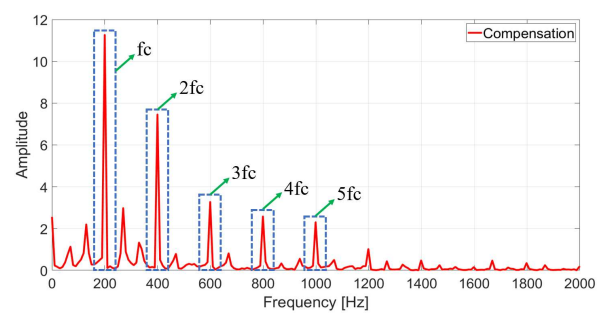

(b)

Fig. 8: Spectrum analysis results of the measured milling force after compensation. (a) x direction. (b) y direction.

\subsection{Comparison of milling force identification results}

According to the transfer function in the $\mathrm{x}$ and $\mathrm{y}$ directions obtained by the impact hammer experiment, the acceleration signal measured in the milling experiment is passed through Butterworth low-pass filtering as input. At the same time, the measured milling force after inverse filter compensation is used as a reference standard to verify the effectiveness of the proposed method.

A fixed number of iterations is given to verify the effectiveness of the proposed FSC-LSQR algorithm. Figure 9 shows the error norm of the LSQR algorithm to identify the milling force and the measured milling force after compensation under 100 iterations. Figure 10 shows the residual norm of the FSC-LSQR algorithm under 100 iterations.

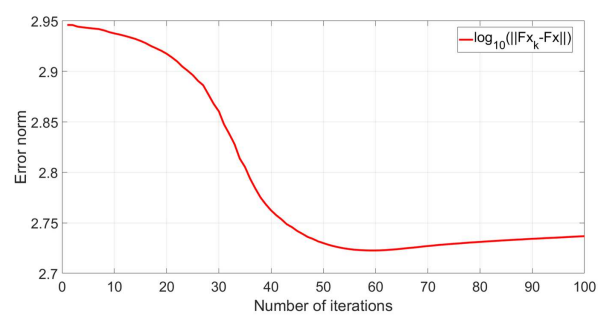

(a)

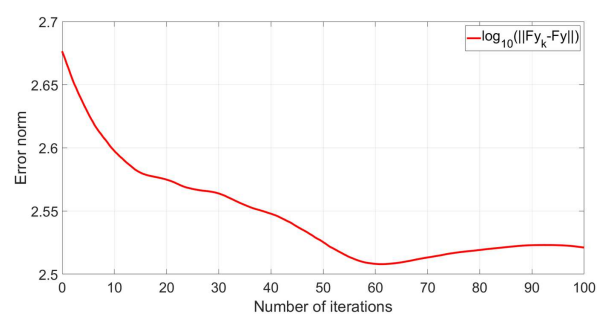

(b)

Fig. 9: Error norm of the LSQR algorithm identify the milling force and the measured milling force after compensation. (a) $\log _{10}\left(\left\|F_{x_{k}}-F_{x}\right\|\right)$ in the $\mathrm{x}$ direction. (b) $\log _{10}\left(\left\|F_{y_{k}}-F_{y}\right\|\right)$ in the y direction. 


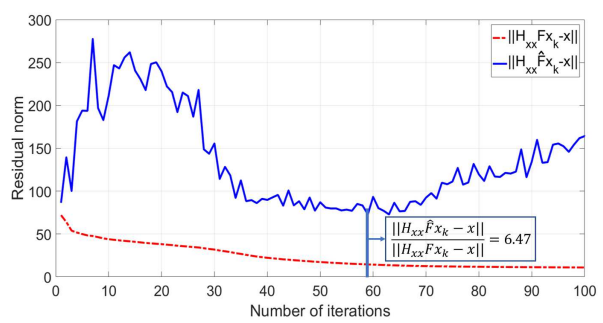

(a)

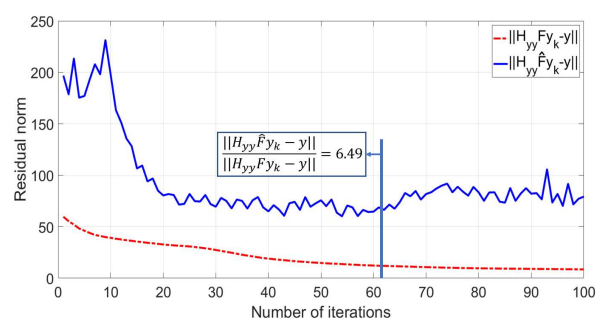

(b)

Fig. 10: Residual norm of the FSC-LSQR algorithm. (a) $\left\|H_{x x} F_{x_{k}}-x\right\|$ and $\left\|H_{x x} \hat{F}_{x_{k}}-x\right\|$ in the $\mathrm{x}$ direction. (b) $\left\|H_{y y} F_{y_{k}}-y\right\|$ and $\left\|H_{y y} \hat{F}_{y_{k}}-y\right\|$ in the $\mathrm{y}$ direction.

As clearly shown in Figures 9 and 10 that if only the LSQR algorithm is used, the error norm first decreases rapidly and then slowly increases as the number of iterations increases, which verifies that the error norm $\left(\left\|F_{x_{k}}-F_{x}\right\|\right.$ and $\left.\left\|F_{y_{k}}-F_{y}\right\|\right)$ of the approximate solution mentioned above does not necessarily satisfy monotonically decreasing. The residual norm $\left(\left\|r_{x_{k}}\right\|=\left\|H_{x x} F_{x_{k}}-x\right\|\right.$ and $\left.\left\|r_{y_{k}}\right\|=\left\|H_{y y} F_{y_{k}}-y\right\|\right)$ satisfies the inequality (10) that $\left\|r_{x_{k}}\right\| \geq\left\|r_{x_{k+1}}\right\|$ and $\left\|r_{y_{k}}\right\| \geq\left\|r_{y_{k+1}}\right\|$. In the actual milling process, it is impossible to equip each machine tool with the reference value of the milling force provided by the Kistler dynamometer to obtain the optimal number of iterations. Morigi et al. [24] seemed to obtain a satisfactory approximate solution and its stopping criterion by finding the local minimum points of $\left\|\boldsymbol{F}_{k+1}-\boldsymbol{F}_{k}\right\|$. However, it is pointed out in the [31] that the effect of this algorithm in numerical experiments is not very well.

As mentioned in Section 3.1, both the LSQR algorithm and the Craig algorithm are based on the Golub-Kahan bidiagonalization process, so the solution speed of the FSC-LSQR algorithm is almost the same as that of the LSQR algorithm in each iteration. In general, the $\left\|\hat{r}_{x_{k}}\right\|$ and $\left\|\hat{r}_{y_{k}}\right\|$ obtained by the Craig algorithm will be greater than the $\left\|r_{x_{k}}\right\|$ and $\left\|r_{y_{k}}\right\|$ obtained by the LSQR algorithm. Hanke. [28] have given some numerical verifications on this property. We determine the value of parameter $\delta$ in the FSC-LSQR algorithm through experiments. It can be seen from Figure 10 that when the residual norm ratios in the $\mathrm{x}$ and $\mathrm{y}$ directions are $\frac{\left\|H_{x x} \hat{F}_{x_{k}}-x\right\|}{\left\|H_{x x} F_{x_{k}}-x\right\|}=6.47$ and $\frac{\left\|H_{y y} \hat{F}_{y_{k}}-y\right\|}{\left\|H_{y y} F_{y_{k}}-y\right\|}=$ 6.49 , respectively, the speed and accuracy of the identified milling force are optimal. The iteration stopping parameter $\delta$ of the FSC-LSQR algorithm is set to 6.5 .

Remark 2: The advantages of the FSC-LSQR algorithm in theory and practical applications are summarized as follows: (1) In theory, the FSC-LSQR algorithm gives the iterative stopping criterion of the optimal solution, so that the milling force can be identified quickly and accurately. (2) In practical applications, the FSC-LSQR algorithm does not need to use the dynamometer 
result as the reference to give the iterative stopping criterion, and the optimal solution of the FSC-LSQR algorithm can be obtained only by using the accelerometer.

The measured acceleration signals are low-pass filtered, and then the obtained signal is used as the input of the algorithm for milling force identification. The results obtained by using the FSC-LSQR and the CGLS algorithms to identify the milling force are shown in Figures 11 and 12.

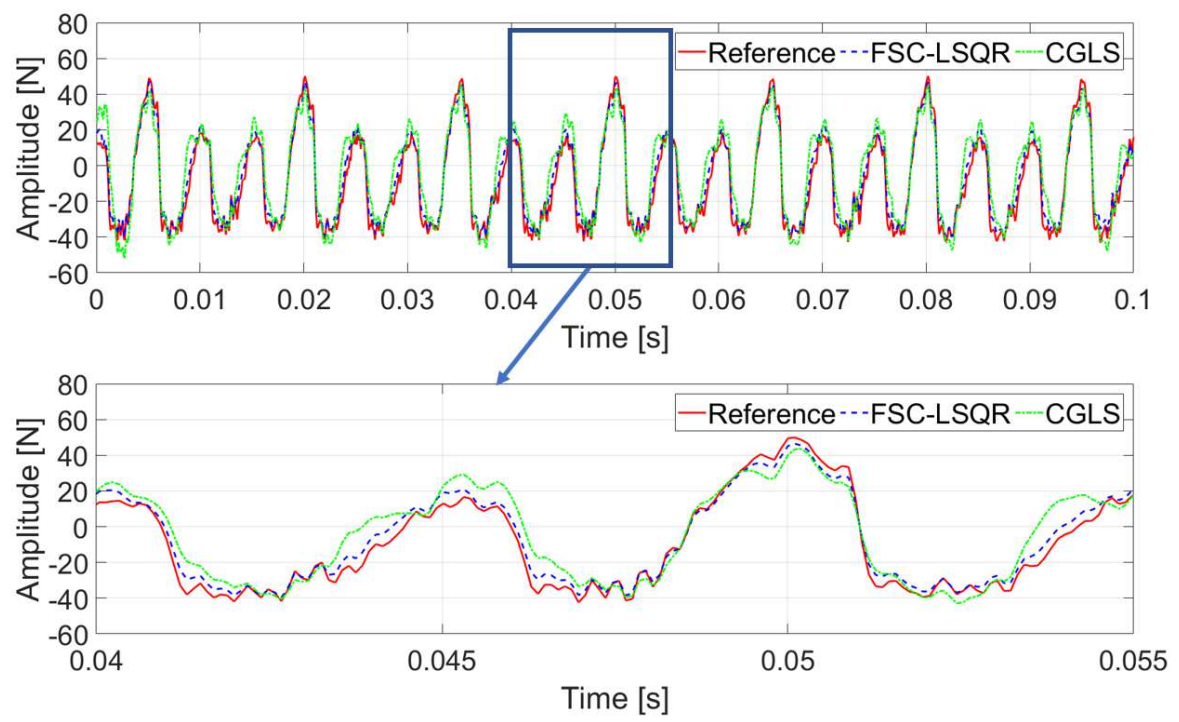

Fig. 11: The measured milling force after compensation and identified milling forces with radical milling depth $1 \mathrm{~mm}$ and speed is $4000 \mathrm{r} / \mathrm{min}$ in the $\mathrm{x}$ direction. 


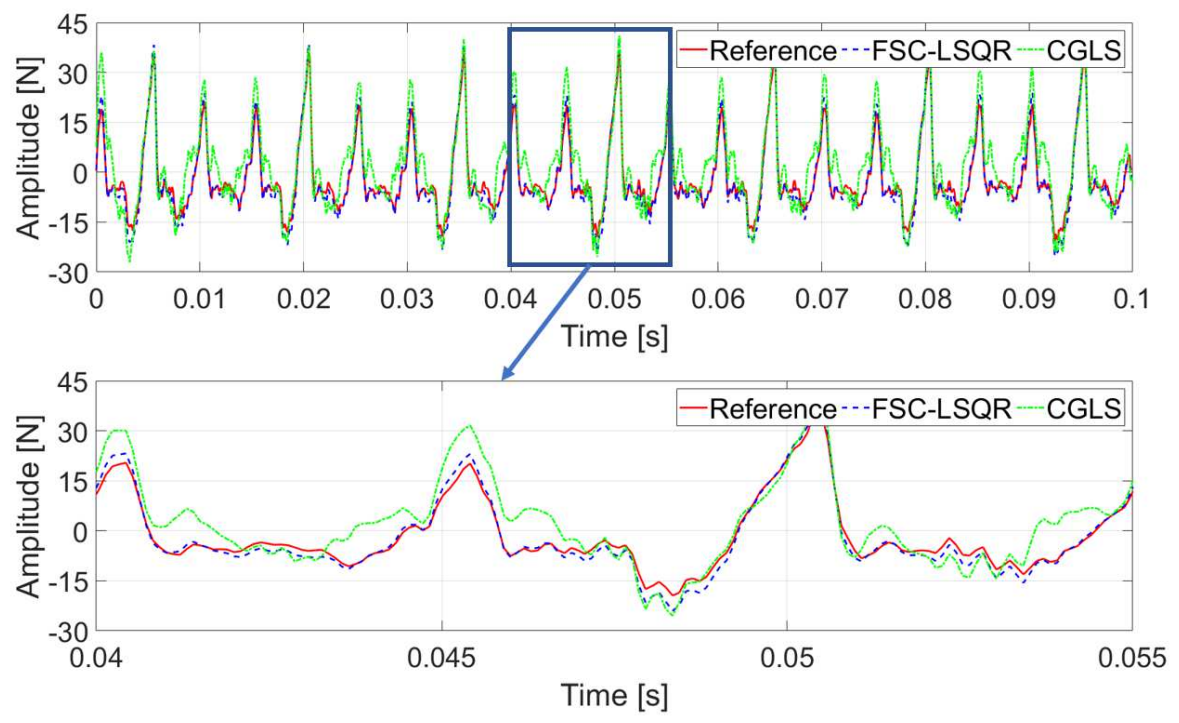

Fig. 12: The measured milling force after compensation and identified milling forces with radical milling depth $1 \mathrm{~mm}$ and speed is $4000 \mathrm{r} / \mathrm{min}$ in the $\mathrm{y}$ direction.

Obviously seen from Figures 11 and 12 that the milling force identified by the the FSC-LSQR algorithm is in good agreement with the measured milling force after compensation. There is a large error between the milling force identified by the CGLS algorithm and the measured milling force after compensation. From the enlarged view, there is still a certain difference between the milling force identified by the FSC-LSQR algorithm and the measured milling force after compensation, which is mainly affected by the complicated path of the acceleration signal from the tool tip to the spindle box and the nonlinearity of the system.

Remark 3: Wang et al. [23] has proved that the accuracy and speed of the CGLS algorithm to identify milling force are higher than the Tikhonov regularization algorithm [32], so we only compare with the CGLS algorithm.

The absolute error between the measured milling force after compensation and the identified milling force in the $\mathrm{x}$ and $\mathrm{y}$ directions is shown in Figure 13, which illustrates that the milling force identified by the FSC-LSQR algorithm has less absolute error than the milling force identified by the CGLS algorithm. 


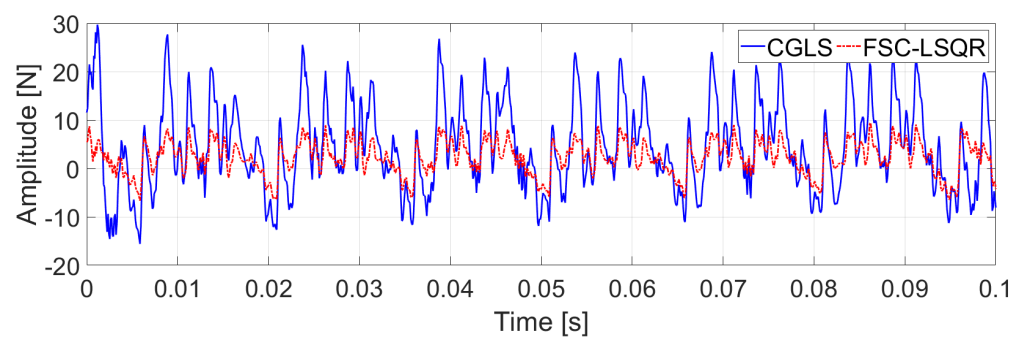

(a)

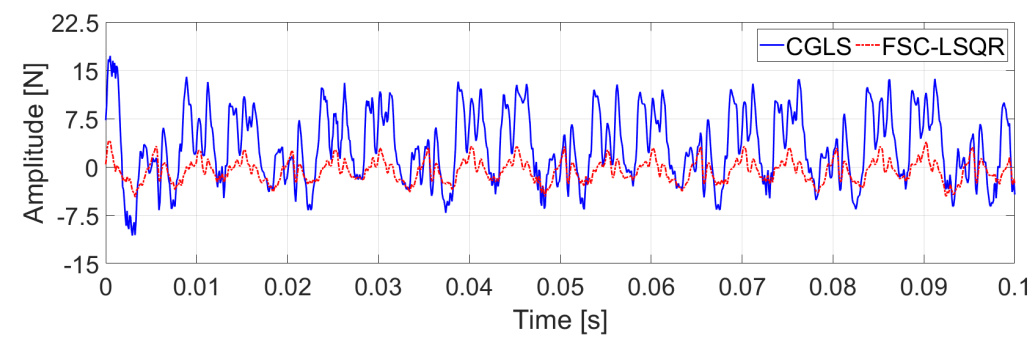

(b)

Fig. 13: The absolute errors between the measured milling force after compensation and the identified milling force. (a) x direction. (b) y direction.

Quantitative analysis of milling force identification based on CGLS and FSC-LSQR algorithms is carried out. The most commonly evaluation indexes (peak-to-peak value and root mean square value) are used to give the evaluation standard for accuracy of milling force identification.

$$
\begin{gathered}
V_{p p}=\frac{\sum_{i=1}^{N}\left|P_{i}^{c p}-P_{i}^{i d}\right|}{\sum_{i=1}^{N} P_{i}^{c p}} \times 100 \% \\
V_{r m s}=\frac{\sum_{i=1}^{N}\left|R_{i}^{c p}-R_{i}^{i d}\right|}{\sum_{i=1}^{N} R_{i}^{c p}} \times 100 \%
\end{gathered}
$$

where $N$ is the number of rotation cycles, $P_{i}^{c p}$ and $P_{i}^{i d}$ are the peak-to-peak value of the measured milling force after compensation and the identified milling force for the $i$-th rotation cycle, $R_{i}^{c p}$ and $R_{i}^{i d}$ are the root mean square value of the measured milling force after compensation and the identified milling force for the $i$-th rotation cycle.

The CGLS and FSC-LSQR algorithms are run in MATLAB R2018a on the same computer to identify the milling force. CPU is AMD Ryzen 7 3700X 8Core Processor $3.59 \mathrm{GHz}$, RAM is $16 \mathrm{G}$, and the operating system is windows 10. Note that the quantitative analysis of the ten cycles milling force is very 
meaningful, the online identification strategy of milling force given by the QBS in Section 3.2 is to take the acceleration signal of ten cycles as an example. In addition, only the milling force of a complete rotation cycle can obtain additional information, such as tool breakage, work path deviation, etc. The results of the quantitative analysis are shown in Figure 14.

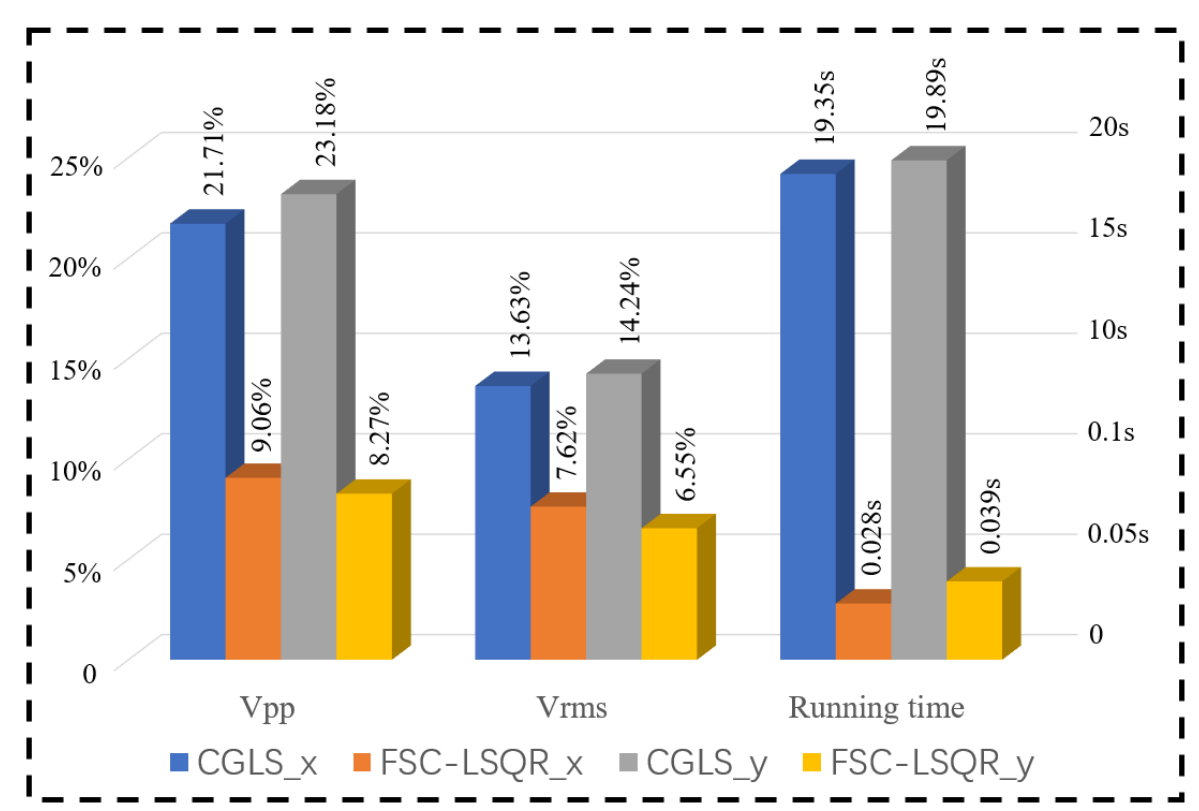

Fig. 14: Quantitative analysis of FSC-LSQR and CGLS algorithms.

It can be seen from Figure 14 that the FSC-LSQR algorithm is superior to the CGLS algorithm in terms of both the peak-to-peak evaluation index $\left(V_{p p}\right)$ and the root mean square evaluation index $\left(V_{r m s}\right)$. The most important thing is that the running time of the FSC-LSQR algorithm is much lower than that of the CGLS algorithm, which provides the possibility of online identification of milling force.

\subsection{Discussions}

According to the online identification strategy of milling force given in Section 3.2 , if the running time of the algorithm to identify ten cycles of milling force is less than the time of ten cycles of milling force dequeue, the online identification of milling force can be realized. According to the sampling frequency of $10240 \mathrm{~Hz}$, the spindle speed is $4000 \mathrm{r} / \mathrm{min}$, and the output time of 512 points collected in ten cycles is $0.05 \mathrm{~s}$. Figure 14 shows the running time of the milling force identification of ten cycles using FSC-LSQR and CGLS algorithms. Among them, the time of the milling force identification using the 
FSC-LSQR algorithm is less than $0.05 s$. In other words, the proposed FSCLSQR algorithm can realize the online identification of milling force with the sampling frequency of $10240 \mathrm{~Hz}$, which completely satisfy the requirements of the actual milling force measurement.

\section{Conclusions}

This paper proposes an industry-oriented method for measuring the milling force based on FSC-LSQR using acceleration signals. The main conclusions and contributions are as follows: (1) A fast stopping criterion for the LSQR algorithm is proposed, which can only need the most common and mature accelerometer in the industry to obtain the optimal speed and accuracy of milling force identification. (2) Using the queue buffer structure, the requirements of the online identification of milling force on the algorithm running time are given, and then the whole process of the online identification of milling force is described. (3) Milling experiment is done, and the quantitative results show that the error between the milling force identified based on the FSCLSQR algorithm and the milling force generated in the actual milling process is less than $10 \%$. In addition, it is also verified that the FSC-LSQR algorithm running time is short enough to realize online identification of milling force.

Furthermore, industrial robots are different from CNC machine tools, that is, the frequency response function of industrial robots from tool tip to spindle box accelerometer will change in the machining process. We will focus on the identification of milling force in the machining process of industrial robots. The ultimate purpose is to embed the accelerometer into the intelligent spindle, so that the intelligent spindle has the ability to online identification of milling force.

\section{Declarations}

Funding: This work is supported by National Natural Science Foundation of China (No. 51922084). Conflicts of interest: The authors would like to declare no competing financial interests or personal relationship that could have influenced the research result reported in this paper. Availability of data and material: It can be obtained from the author by mail. Code availability: Not applicable. Ethics approval: Not applicable. Consent to participate: Not applicable. Consent for publication: Not applicable. Authors' contributions: Maxiao Hou: Conceptualization, Methodology, Writing - original draft, Software. Hongrui Cao: Conceptualization, Methodology, Writing - review \& editing, Supervision, Project administration, Funding acquisition. Qi Li: Resources, Methodology. Jianghai Shi: Conceptualization, Writing - review \& editing. 


\section{References}

[1] Cao HR, Zhang XW, Chen XF, The concept and progress of intelligent spindles: A review, International Journal of Machine Tools and Manufacture. 112 (2017) 21-52, https://doi.org/10.1016/j.ijmachtools.2016.10. 005 .

[2] Wang B, Liu ZQ, Cai YK, et al, Advancements in material removal mechanism and surface integrity of high speed metal cutting: A review, International Journal of Machine Tools and Manufacture. 166 (2021) 103744, https://doi.org/10.1016/j.ijmachtools.2021.103744.

[3] Feng J, Sun Z, Jiang Z, Yang L, Identification of Chatter in Milling of Ti-6Al-4V Titanium Alloy Thin-Walled Workpieces Based on Cutting Force Signals and Surface Topography, International Journal of Advanced Manufacturing Technology. 82 (9-12) (2016) 1909-1920, https: //doi.org/10.1007/s00170-015-7509-0.

[4] Jullien-Corrigan A, Ahmadi K, Measurement of high-frequency milling forces using piezoelectric dynamometers with dynamic compensation, Precision Engineering. 66 (2020) 1-9, https://doi.org/10.1016/j.precisioneng. 2020.07.001.

[5] Rizal M, Ghani JA, Nuawi MZ, et al, Development and testing of an integrated rotating dynamometer on tool holder for milling process, Mechanical Systems and Signal Processing. 52 (2015) 559-576, https: //doi.org/10.1016/j.ymssp.2014.07.017.

[6] Jeong YH, Cho DW, Estimating cutting force from rotating and stationary feed motor currents on a milling machine, International Journal of Machine Tools and Manufacture. 42 (2002) 1559-1566, https://doi.org/ 10.1016/S0890-6955(02)00082-2.

[7] Aslan D, Altintas Y, Prediction of Cutting Forces in Five-Axis Milling Using Feed Drive Current Measurements, IEEE/ASME Transactions on Mechatronics.23 (2) (2018) 833-844, https://doi.org/10.1109/TMECH. 2018.2804859 .

[8] Kim D, Jeon D, Fuzzy-logic control of cutting forces in CNC milling processes using motor currents as indirect force sensors, Precision Engineering. 35 (2011) 143-152, https://doi.org/10.1016/j.precisioneng.2010. 09.001 .

[9] Chen YL, Tao Y, Hu P, et al, Self-sensing of cutting forces in diamond cutting by utilizing a voice coil motor-driven fast tool servo, Precision Engineering. 71 (2021) 178-186, https://doi.org/10.1016/j.precisioneng. 2021.03.009. 
[10] Song KY, Wang M, Liu LM, et al, Intelligent recognition of milling cutter wear state with cutting parameter independence based on deep learning of spindle current clutter signal, The International Journal of Advanced Manufacturing Technology. 109 (2020) 238-243, https://doi.org/10.1007/ s00170-020-05587-1.

[11] Castro LR, Vieville P, Lipinski P, Correction of dynamic effects on force measurements made with piezoelectric dynamometers, International Journal of Machine Tools and Manufacture. 46 (14) (2006) 1707-1715, https://doi.org/10.1016/j.ijmachtools.2005.12.006.

[12] Kim JH, Chang DC, Jang DY, Cutting Force Estimation by Measuring Spindle Displacement in Milling Process, CIRP Annals. 54 (1) (2005) 67-70, https://doi.org/10.1016/S0007-8506(07)60051-1.

[13] Kim LH, Dynamic cutting force on-line estimation using a 4-electrode cylindrical capacitive displacement sensor mounted on a high speed milling spindle, Journal of Mechanical Science and Technology. 22 (2008) 914-923, https://doi.org/10.1007/s12206-008-0214-2.

[14] Albrecht A, Park SS, Altintas Y, et al, High frequency bandwidth cutting force measurement in milling using capacitance displacement sensors, International Journal of Machine Tools and Manufacture. 45(9) (2005) 993-1008, https://doi.org/10.1016/j.ijmachtools.2004.11.028.

[15] Zhou J, Mao XY, Liu HQ, et al, Prediction of cutting force in milling process using vibration signals of machine tool, The International Journal of Advanced Manufacturing Technology. 99 (2018) 965-984, https://doi. org/10.1007/s00170-018-2464-1.

[16] Salehi M, Albertelli P, Goletti M, et al, Indirect Model Based Estimation of Cutting Force and Tool Tip Vibrational Behavior in Milling Machines by Sensor Fusion, Procedia CRIP. 33 (2015) 239-244, https://doi.org/10. 1016/j.procir.2015.06.043.

[17] Hamid M, Chaneel IP, Guseon K, et al, Reconstruction of cutting forces through fusion of accelerometer and spindle current signals, Journal of Manufacturing Processes. 68 (2021) 990-1003, https://doi.org/10.1016/j. jmapro.2021.06.007.

[18] Spiewak SA, Acceleration based indirect force measurement in metal cutting processes, International Journal of Machine Tools and Manufacture. 35 (1) (1995) 1-17, https://doi.org/10.1016/0890-6955(95)80005-0.

[19] Korkmaz E, Gozen BA, Bediz B, Ozdoganlar OB, Accurate measurement of micromachining forces through dynamic compensation of dynamometers, Precision Engineering. 49 (2017) 365-376, https://doi.org/10.1016/ 
j.precisioneng.2017.03.006.

[20] Chae J, Park SS, High frequency bandwidth measurements of micro cutting forces, International Journal of Machine Tools and Manufacture. 47 (9) (2007) 1433-1441, https://doi.org/10.1016/j.ijmachtools.2006.09.028.

[21] Pawelko P, Powalka B, Berczynski, Estimation of Cutting Force Model Coefficients with Regularized Inverse Problem, Advances in Manufacturing Science and Technology. 37 (2) (2013) 5-21, https://doi.org/10.2478/ amst-2013-0012.

[22] Wang CX, Zhang XW, Qiao BJ, et al, Milling force identification from acceleration signals using regularization method based on TSVD in peripheral milling, Procedia CIRP. 77 (2018) 18-21, https://doi.org/10. 1016/j.procir.2018.08.195.

[23] Wang CX, Zhang XW, Qiao BJ, et al, Dynamic Force Identification in Peripheral Milling Based on CGLS Using Filtered Acceleration Signals and Averaged Transfer Functions, Journal of Manufacturing Science and Engineering. 141 (6) (2019) 064501, https://doi.org/10.1115/1.4043362.

[24] Morigi S, Reichel L, Sgallari F, et al, Iterative methods for ill-posed problems and semi-convergent sequences, Journal of Computational and Applied Mathematics. 193 (2006) 157-167, https://doi.org/10.1016/j.cam. 2005.05.028.

[25] Rao SS and Yap FF, Mechanical Vibrations (5th Edition). (2011), https: //doi.org/10.1017/S0001924000067099.

[26] Meurant G, The Lanczos and Conjugate Gradient Algorithms, SIAM, Philadelphia. (2006), https://doi.org/10.1017/S096249290626001X.

[27] Jia ZX, Regularization properties of LSQR for linear discrete ill-posed problems in the multiple singular value case and best, near best and general low rank approximations, Inverse Problems. 36 (2020) 085009, https://doi.org/10.1088/1361-6420/ab9c45.

[28] Hanke M, On Lanczos Based Methods for the Regularization of Discrete Ill-Posed Problems, BIT Numerical Mathematics. 41 (2001) 1008-1018, https://doi.org/10.1023/A:1021941328858.

[29] Gupta GK, Banerjee A, On Finite Buffer Bulk Arrival Bulk Service Queue with Queue Length and Batch Size Dependent Service, International Journal of Applied and Computational Mathematics. 5 (2) (2019), https://doi.org/10.1007/s40819-019-0617-z. 
[30] Wan M, Yin W, Zhang WH, et al, Improved inverse filter for the correction of distorted measured cutting forces, The International Journal of Mechanical Sciences. 120 (2017) 276-285, https://doi.org/10.1016/j. ijmecsci.2016.11.033.

[31] Bauer F, Kindermann S, The quasi-optimality criterion for classical inverse problems, Inverse Problems. (2008), 035002, https://doi.org/10. 1088/0266-5611/24/3/035002.

[32] Park Y, Reichel L, Rodriguez G, et al, Parameter determination for Tikhonov regularization problems in general form, Journal of Computational and Applied Mathematics. 343 (1) (2018) 12-25, https://doi.org/ 10.1016/j.cam.2018.04.049. 